\title{
MAPA DE NIVELES SONOROS URBANOS DEL MICROCENTRO DE LA CIUDAD DE POSADAS, MISIONES.
}

Ing. Traid, Hernán D.; Mg. Puchalski, Cecilia M.; Morinisi, Rodrigo S.; Mg. Basaldua, Carlos A.; Mg. Jejer, Carlos S. (*)

\section{RESUMEN}

Un problema ambiental que aqueja actualmente a las zonas urbanas contemporáneas es la contaminación sonora. En aras de caracterizar los niveles sonoros en el microcentro de la ciudad de Posadas, se llevaron a cabo mediciones de 15 minutos en banda de tercio de octavas $1 / 3$, ponderación A y respuesta temporal rápida, registrando un dato como nivel sonoro equivalente $\left(\mathrm{L}_{\mathrm{eq}}\right)$ cada $3 \mathrm{~s}$. El instrumento se ubicó en la vereda a una altura de $1,5 \mathrm{~m}$ y a mitad de cuadra, en 71 puntos distribuidos representativamente en el área de estudio. Los datos obtenidos permitieron la confección del mapa de niveles sonoros. Se halló diferencias entre las calles con y sin transporte público y al contrastar los resultados con la normativa municipal vigente, se determinó que en el $20 \%$ de los puntos los niveles sonoros alcanzaron el "nivel de precaución".

Palabras Clave: niveles sonoros, Posadas, mapa de niveles sonoros, ruido urbano.

\section{INTRODUCCIÓN}

El crecimiento de una ciudad trae aparejado, como una consecuencia indeseada e inevitable, el incremento de niveles sonoros ambientales a valores tales, que pueden tornarse nocivos para los habitantes. Según la definición de la Real Academia Española (2013), el ruido es un sonido percibido como desagradable. Puede alterar nuestras actividades diarias, dificultar la comunicación, generando molestias y trastornos del sueño, además de una precoz pérdida de audición (GCBA, 2008).

La ciudad de Posadas ha crecido notablemente en los últimos años, registrando un incremento poblacional del 14,24 \% en el último decenio (IPEC, 2011), y hasta la fecha no se han encontrado antecedentes de evaluaciones sistemáticas de los niveles sonoros urbanos. Es menester que instituciones como la Universidad Nacional de Misiones asuma el compromiso para con la sociedad, de dar los primeros pasos en evaluar temas de interés general relacionados al medio ambiente y la calidad de vida. 
El área geográfica escogida para abordar la problemática fue el casco céntrico de la ciudad de Posadas, comprendida entre las avenidas: Comandante Andresito Guaçurarí, Corrientes, Bartolomé Mitre y Roque Sáenz Peña; con una superficie aproximada de $2 \mathrm{~km} 2$. Es un área urbana de media y alta densidad destinada a la consolidación y localización del equipamiento administrativo, comercial, financiero e institucional a escala regional y urbana, con usos diversos que coexisten con el uso residencial (Municipalidad de Posadas, 2011). Esto se pone de manifiesto en la zonificación establecida por el Código de Planeamiento Urbano. En ella, "C" corresponde a Distritos Centrales (incluye actividades comerciales), " $\mathrm{R}$ " a Distritos Residenciales, "AE" a Distritos de Arquitectura Especial y "U" a Distrito Urbanización Determinada (Figura 1)

En una etapa preliminar se realizó un monitoreo de 24 horas en 15 puntos del microcentro de la ciudad de Posadas, a fin de caracterizar los niveles sonoros urbanos (Figura 1).

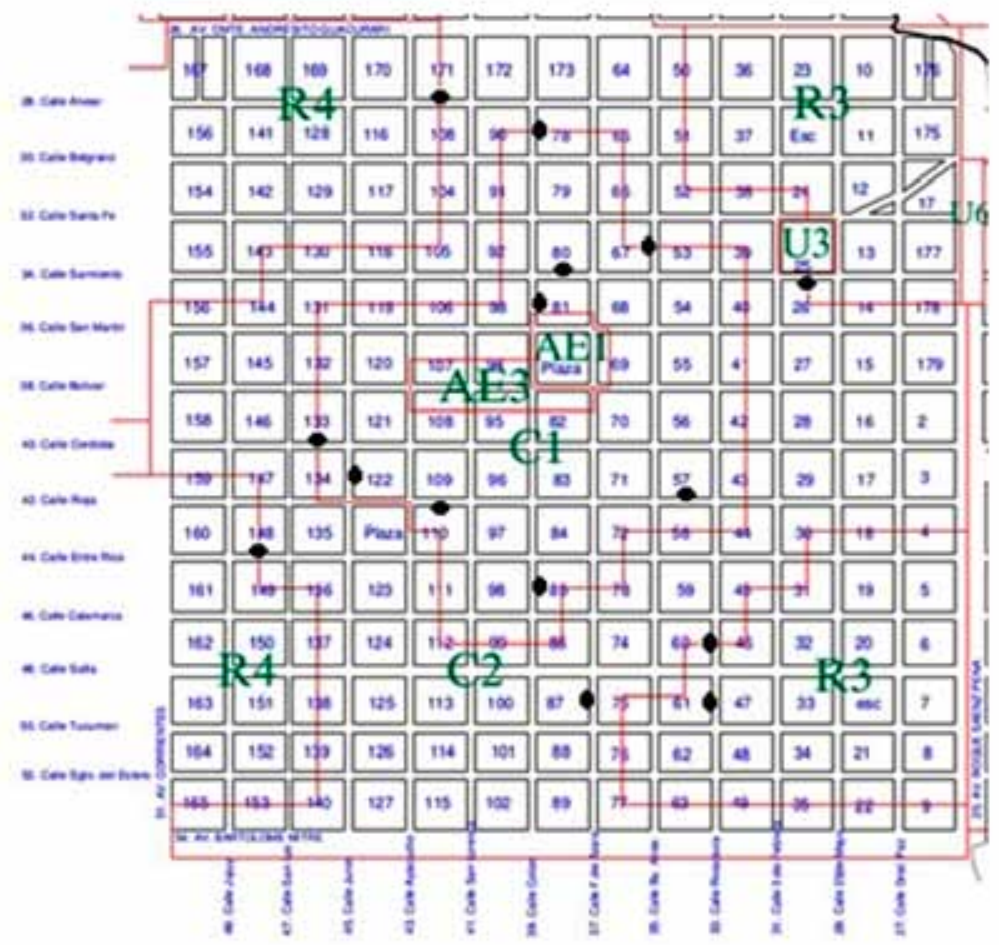

Figura 1. Zonas según Código de Planeamiento Urbano y puntos de mediciones exploratorias. Fuente: Municipalidad de Posadas. Recuperado de: http://www.ordenamiento.posadas.gov.ar/index.php

Las mediciones se realizaron en días hábiles, utilizando ponderación A, la cual representa mejor la percepción del sonido por el ser humano, con banda de octavas $1 / 1$ y respuesta rápida. Se adquirió un dato integrado cada 30 segundos, registrándolo como nivel sonoro equivalente $\left(\mathrm{L}_{\mathrm{eq}}\right)$ en $\mathrm{dB}(\mathrm{A})$. $\mathrm{El}$ instrumento se ubicó a 4 metros del suelo, a 2 metros de la calle (medidos horizontalmente) y equidistante entre bocacalles (mitad de cuadra), obteniendo valores de 15 puntos distribuidos con la mayor representatividad posible dentro del área de estudio, bajo consideraciones de disponibilidad de espacio para la instalación del equipamiento y condiciones propicias para la preservación 
del mismo, tanto por inclemencias climáticas como de vandalismo (Miyara, 2003).

A través del análisis de los resultados obtenidos, fue posible determinar las franjas horarias de mayor representatividad y brindar sustento a la metodología elaborada y empleada en el presente trabajo (Morinisi y col., 2015).

La actual investigación tiene por objetivo aplicar realizar una caracterización de los niveles sonoros urbanos existentes en el microcentro de la ciudad de Posadas mediante la construcción de un mapa de niveles sonoros basado en metodologías desarrolladas específicamente para el área de estudio y cotejar los resultados con la normativa vigente.

\section{METODOLOGIA}

\section{Grilla de muestreo}

En la ciudad de Posadas y en Argentina, actualmente no existe legislación que especifique una metodología estandarizada para la realización de medición de niveles sonoros urbanos.

La norma IRAM 4113-2 (vigente) para Descripción, Medición y Evaluación del Ruido Ambiental, sólo especifica: "Para el trazado de mapas, los niveles de ruido en los distintos puntos de la grilla de medición normalmente son calculados.[...] En general, la diferencia en niveles de presión sonora entre puntos de la red adyacentes no de ser mayor a $5 d B$. Si se encuentran diferencias significativamente mayores, deben agregarse puntos intermedios en la cuadricula".

A fin de garantizar que la densidad de puntos adoptada sea tal que cumpla con lo especificado en la norma, para la definición del número de puntos de la grilla se consideran dos criterios, uno basado en la experiencia de los modelos geoestadísticos, que considera que el número mínimo necesario de puntos para representar adecuadamente la distribución espacial de una variable determinada es 50 (Chilés y col., 1999). El otro criterio parte de la delimitación geográfica del área objeto de estudio, a partir de la extensión del área se define un número de cuadrículas regulares para realizar la evaluación en cada una.

La técnica de retícula es la forma más directa de obtener los datos que permitan definir las condiciones de contorno de los mapas. La selección del tamaño de la retícula influye enormemente en el número de puntos a medir, y por ende, en el costo del trabajo. Para el presente estudio, se ha utilizado una cuadrícula bastante densa, acorde con el área urbana y los fines de la investigación

Así, para la definición de la retícula y la selección de los puntos de muestreo se ha adoptado una metodología mixta reticularvial (Barrigón y col., 1999; García, 2002; Ling, 1997; Suárez, 2002; Viro y col., 2002). En principio (metodología reticular), el área se dividió en una retícula de $100 \mathrm{~m} \times 100 \mathrm{~m}$, ubicando los puntos a mitad de cada cuadra en todas las cuadras del área de estudio totalizando 406 puntos iniciales y en segunda instancia (metodología vial), se distribuyeron los puntos de medición aleatoriamente en el área de estudio, asignándose dos puntos por cada calle del casco céntrico y agregando un punto adicional a aquellas calles en las que circula el servicio urbano de colectivos, avenidas que comprenden el contorno del área de estudio y las calles con características particulares (paseos y peatonales)

De esta manera, la grilla de muestreo quedó conformada por 71 puntos representativos que permiten caracterizar de manera 
adecuada cada uno de los diferentes tipos de calles/avenidas que se encuentran en el casco céntrico, siendo además consistente con las metodologías y las experiencias realizadas en otras ciudades del país y del mundo.

\section{Descriptor}

El nivel sonoro continuo equivalente (Leq), se conoce en todo el mundo como el parámetro promedio esencial y se mide directamente con un sonómetro integrador. Es una medida de la energía promedio en un nivel sonoro variante, no siendo una medida directa de la molestia. Sin embargo, investigaciones más amplias, han mostrado que el $\mathrm{L}_{\mathrm{eq}}$ se correlaciona bien con la molestia, aunque es evidente que un nivel de ruido aceptable, por ejemplo, un miércoles por la tarde, podría ser angustiante un domingo temprano por la mañana (Bruel y col., 2000).

\section{Ponderación}

La ponderación A fue recomendada por la EPA para describir el ruido medioambiental, debido a que se ajusta a la respuesta del oído humano, es exacta para muchos propósitos y es utilizada mundialmente (Harris, 1995). Así, la intensidad del sonido fue medida en niveles de presión acústica ponderados $\mathrm{A} o \mathrm{~dB}(\mathrm{~A})$, que es la unidad estandarizada para medir el ruido a los efectos de compatibilizar y permitir comparaciones en el ámbito internacional.

En relación a la velocidad de respuesta, las mediciones se realizaron utilizando la velocidad de respuesta Rápida -que corresponde a un intervalo de tiempo de $125 \mathrm{~ms}^{-}$ya que se correlaciona mejor con la percepción humana (IRAM) ${ }^{1}$.

\section{Tiempo de medición}

Si bien los tiempo de medición hallados en la bibliografía se halla dispersa (de 2 minutos por hora hasta mediciones de 24 horas), lo cierto es que cuanto menor sea el tiempo de muestreo mayor será la desviación estándar resultante en los valores de $\mathrm{L}_{\text {eq }}$, es decir, si se mide un minuto por cada hora, la desviación estándar de los Leq horarios será mayor que si medimos 30 minutos por hora. En diversos estudios, se ha determinado que una buena solución de compromiso entre el desvío de la medición y carga horaria de los recursos empleados es realizar mediciones de 15 minutos, siendo que una medición más extensa no aporta mucha más información e incrementa sensiblemente los costos (Viro y col., 2002). Esto ha quedado plasmado en la redacción de la Ordenanza Municipal VI - No 14 que requiere que la medición del ruido del tráfico sea de 15 minutos.

Del análisis de los datos del estudio preliminar se desprende que los valores de $\mathrm{L}_{\mathrm{eq}} 15 \mathrm{~min}$ en las franjas horarias de 7 a $11 \mathrm{~h}$ y de 17 a $20 \mathrm{~h}$ presentan una desviación menor o igual a $2 \mathrm{~dB}(\mathrm{~A})$ para al menos el $70 \%$ de los datos respecto al Leq,24h siendo estas las ventanas horarias de mayor representatividad.

Por otra parte, para la elaboración del mapa de ruido de la ciudad, se tuvo en cuenta que "para evaluaciones a largo plazo y planificación del uso del suelo, deberían usarse los intervalos de tiempo a largo plazo que representan alguna fracción significativa de un año (por ejemplo, 3 meses, 6 meses, 1 año)" 2. Teniendo en cuenta este requisito y las características de la ciudad descritas anterior-

${ }^{1}$ IRAM 4113-2, Sección 8.4.5.

${ }^{2}$ IRAM 4113-1. Sección 8.1 
mente, las mediciones se realizaron en los meses de marzo a noviembre, con excepción de las semanas de las vacaciones de julio.

Finalmente, se adquirió un dato integrado cada tres segundos, registrado el valor como nivel sonoro equivalente $\left(\mathrm{L}_{\mathrm{eq}}\right)$.

\section{Equipamiento}

Para la realización de las mediciones se empleó un decibelímetro marca TES1358, con analizador de banda octavas $1 / 1$ y $1 / 3$; Clase 2 y acorde a IRAM 4081; IEC 60651/804 tipo 2; ANSI S1.4 tipo 2; calibrador marca TES-1356 (calibrado anualmente) que es el correspondiente al decibelímetro empleado; pantalla antiviento integrada, trípode; baterías y; pc con software/interfaz para descarga de datos.

El equipamiento se instaló sobre la vereda, a una altura de $1,5 \mathrm{~m}$, orientado hacia la calzada, evitando la proximidad de cualquier objeto reflectante, y a mitad de cuadra, para evitar la influencia de los semáforos, las paradas de colecti- vos y que el valor registrado sea el resultado del sonido proveniente de dos vías.

\section{Registro de la información}

La planilla de campo fue confeccionada atendiendo los requerimientos de las normativas vigentes, entre las cuales se encuentran la Ordenanza VI - No 14 y la norma IRAM 4113. La información registrada en cada punto de muestreo fue la siguiente:

- Fecha, hora y lugar de la medición

- Sitio de medición: ubicaciones de micrófono (altura y posición) y fuente.

- Condiciones operativas: Cantidad y tipo de vehículos que circulan.

- Condiciones meteorológicas: velocidad y dirección del viento, temperatura, presión barométrica, humedad.

- Eventos particulares durante la medición (alarmas, gritos, congestión en el tráfico que derive en la

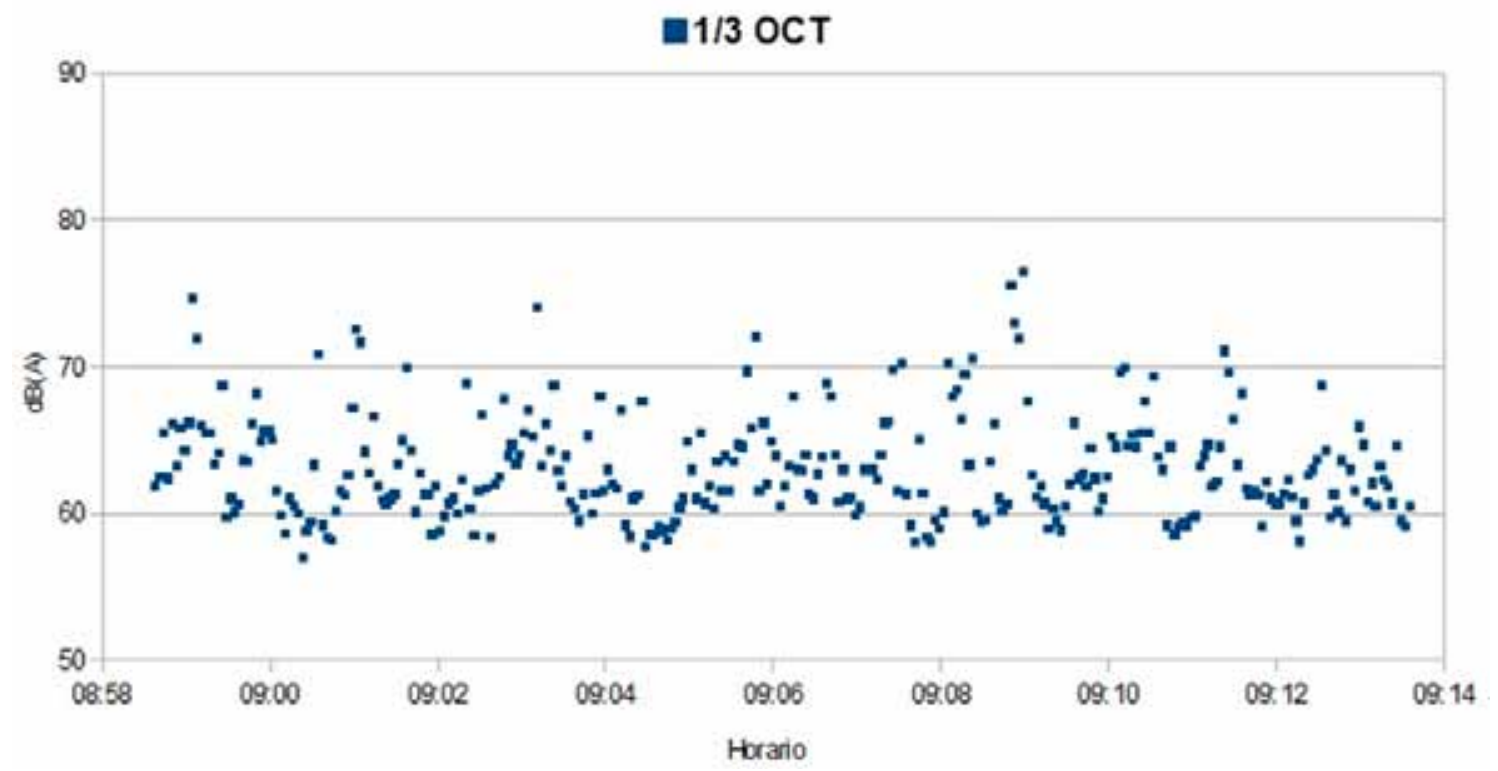

Figura 2. Perfil de niveles sonoros para una medición. 
utilización repetida del claxon, etc.)

En primera instancia, se elaboraron los perfiles temporales de los niveles sonoros en cada uno de los puntos de medición. Estas gráficas resultan útiles ya que permiten analizar la influencia de los acontecimientos ordinarios y fortuitos observados durante la obtención de los datos que, integrados, conformarán el valor del nivel sonoro equivalente $\mathrm{L}_{\text {eq, } 15 \mathrm{~min} \text {. }}$
En el caso presentado en la Figura 2 se aprecia una oscilación que es característica de aquellas calles que poseen semáforos y, además, que no existió ningún evento extraordinario (alarmas, silbidos, etc.) durante el período de medición.

Luego, en base a los valores de $\mathrm{L}_{\text {eq,15min }}$ se elaboró el primer mapa de niveles sonoros urbanos del microcentro de la ciudad de Posadas (Figura 3)

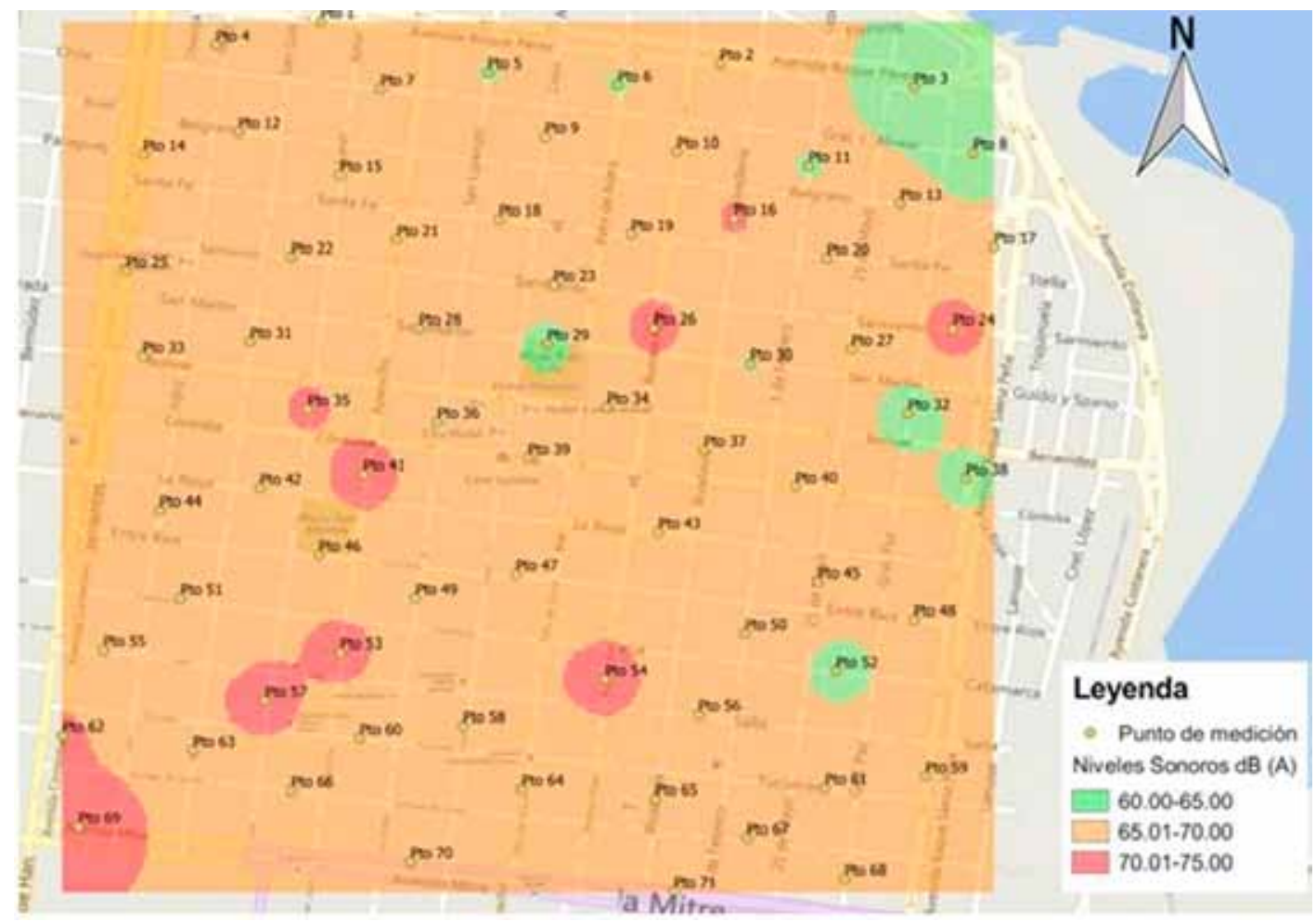

Figura 3. Mapa de niveles sonoros del microcentro de la ciudad de Posadas.

En relación al cumplimiento de la Ordenanza VI - No 14, específicamente, en el capítulo $\mathrm{V}$, art. 13 se refiere a la contaminación por ruido de Responsabilidad Colectiva, considerando "al tránsito vehicular como una fuente de contaminación por ruido con entidad propia de carácter colectivo y no punible [...] cuando el ruido proveniente del tránsito vehicular alcance niveles capaces de comprometer la salud y bienestar público, la Municipalidad está obligada a intervenir con medidas correctivas". Los tipos de intervención estipuladas en la normativa son "tres (3) tipos de intervención, según sea el valor de $L_{e q}$ ". 


\begin{tabular}{|l|l|l|}
\hline \multicolumn{1}{|c|}{$\mathbf{L}_{\text {eq }} \mathbf{d B}(\mathbf{A})$} & \multicolumn{1}{c|}{ Calificación } & \multicolumn{1}{c|}{ Tipo de intervención } \\
\hline 70 o menos & Nivel de seguridad & Ninguna \\
\hline Entre 70 y 75 & Nivel de precaución & $\begin{array}{l}\text { Estado de alerta; monitoreo frecuente y } \\
\text { acción preventiva }\end{array}$ \\
\hline 75 o más & Nivel de acción & Acción correctiva inmediata \\
\hline
\end{tabular}

Tabla 1. Tipos de Intervención según sea el valor del Nivel Sonoro Continuo Equivalente. Fuente: Ordenanza VI - No 14 del Honorable Concejo Deliberante de la ciudad de Posadas.

De los datos obtenidos hasta el momento, 142 mediciones (dos por cada punto de la grilla), se observa que en ningún caso se alcanzó el "Nivel de Acción", esto es un valor superior a un $\mathrm{L}_{\text {eq }}$ de $75 \mathrm{~dB}(\mathrm{~A})$. Sin embargo en el $20 \%$ de los puntos, se alcanzó el "Nivel de Precaución" que por normativa exige la intervención de organismos públicos con la realización de un monitoreo frecuente y medidas de acción preventivas.

Se ha determinado además, que existen diferencias estadísticamente significativas entre las medias de los niveles sonoros observados en las calles por las cuales circula el transporte público y por las que no lo hace ( $\mathrm{p}$-valor $<0.05)$.

\section{CONCLUSIONES}

La metodología definida en la primera etapa del proyecto fue utilizada $y$, en vistas de los resultados obtenidos a la fecha, se concluye preliminarmente que es adecuada para los objetivos planteados. Asimismo, la planilla de campo empleada fue exitosamente adaptada a lo largo de las mediciones para el pertinente registro de elementos de interés, lográndose una ade- cuada caracterización del área de estudio.

Se obtuvo el primer mapa de ruido para el microcentro de la ciudad de Posadas y se continuarán realizando las mediciones hasta dar cumplimiento con la totalidad de las mediciones planificadas.

Al momento, los datos obtenidos indican que en el $20 \%$ de los puntos los niveles sonoros alcanzaron el "nivel de precaución" establecido en la normativa, instando al organismo de aplicación a realizar monitoreos frecuentes y adopción de acciones preventivas. Además se ha verificado que existen diferencias significativas entre el $\mathrm{L}_{\text {eq15min }}$ medido en calles por las que circula el transporte público y el obtenido en las que no lo hace.

Los resultados obtenidos resultan promisorios, siendo los primeros en torno a la problemática de la contaminación sonora urbana en la ciudad de Posadas con un abordaje sistemático. La información generada durante el desarrollo del presente proyecto permitirá establecer medidas de gestión efectivas con basamento científico que permitan mejorar la calidad de vida de residentes y transeúntes del área de estudio. 


\section{BIBLIOGRAFÍA}

- Barrigón J., Gómez V., Gutiérrez, P., Alejandre L., Casillas M. y Ahmed J. (1999). Estudio Preliminar del Ruido Ambiental en la Ciudad de Cáceres. Tecniacústica 1999. Ed. Sociedad Española de Acústica. España.

- Bruel y Kjaer (2000). Ruido $M e^{-}$ diambiental. Brüel \& Kjaer, División of Spectris. España, S.A. 69 pp

- Chilés, J. y Delfiner, P. (1999). Geoestadistics Modeling Spatial uncertainty: Wiley Series In Probability and Statistics. United Sates of America.

- GarcíaA.(2002).RealizacióndeMapasAcústicos. Memorias Jornadas Internacionales sobre Contaminación Acústica en las Ciudades. Mesa Redonda MR.05-1. Madrid.

- GCBA (Gobierno de la Ciudad de Buenos Aires), Agencia de Protección Ambiental. Plan Estratégico 20082012. Buenos Aires. Noviembre 2008.

- Harris, C. M. Manual de Medidas Acústicas y Control del Ruido. Mc Graw Hill. $3^{\circ}$ Edición. 1995. ISBN: 84-481-1619-4.

- Instituto Provincial de Estadística y Censos (IPEC). Más de un millón de vecinos: resultados oficiales del censo del bicentenario en misiones. En http://www. ipecmisiones.com. Misiones. 2011. Fecha de acceso: 13 de febrero de 2013.

- IRAM 4113-1 (2009). Acústica. Descripción, medición y evaluación del ruido ambiental. Parte 1 - Magnitudes básicas y métodos de evaluación.

- IRAM 4113-2 (2009). Acústica. Des- cripción, medición y evaluación del ruido ambiental. Parte 2: Determinación de niveles de ruido ambiental.

- Ling M. (1997). An Introduction to Noise Mapping. Institute of Acoustics Bulletin, Building Research Establishment Ltd.UK.

- Miyara, F. (2003). Mediciones de ruido en exteriores. Biblioteca Virtual del Laboratorio De Acústica Y Electroacústica. Escuela De Ingeniería Electrónica, Facultad De Ciencias Exactas, Ingeniería Y Agrimensura, Universidad Nacional De Rosario. Recuperado de: bttp://www.fceia. unr.edu.ar/acustica/bibliolexterior.pdf

- Morinisi, R. S.; Traid, H. D.; Puchalski, C. M.; Basaldua C.A.y Jejer C. S. 5to Congreso de Ciencias Ambientales. COPIME 2015.

- Municipalidad de Posadas (2011). Plan Urbano Ambiental de Posadas. http://www. ordenamiento.posadas.gov.ar/index.php

- Suárez E. (2002). Metodologías Simplificadas para estudios en Acústica Ambiental: Aplicación en la Isla de Menorca. (Tesis de Doctorado). UniversidadPolitécnicadeMadrid.Madrid.

- TES Electrical Electronic Corporation (2009). TES Sound Analyzer 1358, Instruction Manual. Taiwan.

- Viro, G. E.; Bonello, O. J.; Gavinowich, D. S. y Ruffa, F. (2002) Protocolo de mediciones para trazado de mapas de ruido normalizados. (Tesis de grado). Laboratorio de Acústica y Electroacústica (LACEAC), Facultad de Ingeniería, Universidad de Buenos Aires. Buenos Aires. Recuperado de: http://www.fceia. unr.edu.ar/acustica/biblio/protoc-fiuba.pdf 